\title{
A Dynamic Robust Restoration Framework for Unbalanced Power Distribution Networks
}

\author{
Xu, Junjun ; Wu, Zaijun; Yu, Xinghuo ; Cheng, Sheng; Hu, Qinran; Wu, Qiuwei
}

Published in:

IEEE Transactions on Industrial Informatics

Link to article, DOI:

10.1109/TII.2020.2964796

Publication date:

2020

Document Version

Peer reviewed version

Link back to DTU Orbit

Citation (APA):

Xu, J., Wu, Z., Yu, X., Cheng, S., Hu, Q., \& Wu, Q. (2020). A Dynamic Robust Restoration Framework for Unbalanced Power Distribution Networks. IEEE Transactions on Industrial Informatics, 16(10), 6301 - 6312. https://doi.org/10.1109/TII.2020.2964796

\section{General rights}

Copyright and moral rights for the publications made accessible in the public portal are retained by the authors and/or other copyright owners and it is a condition of accessing publications that users recognise and abide by the legal requirements associated with these rights.

- Users may download and print one copy of any publication from the public portal for the purpose of private study or research.

- You may not further distribute the material or use it for any profit-making activity or commercial gain

- You may freely distribute the URL identifying the publication in the public portal 


\title{
A Dynamic Robust Restoration Framework for Unbalanced Power Distribution Networks
}

\author{
Junjun Xu, Member, IEEE, Zaijun Wu, Member, IEEE, Xinghuo Yu, Fellow, IEEE, Sheng Cheng, \\ Qinran Hu, Member, IEEE and Qiuwei Wu, Senior Member, IEEE
}

\begin{abstract}
The increasing penetration of photovoltaic (PV) generators has led to a reduction in the effectiveness of existing strategies for restoring the power distribution network. This paper proposes a dynamic robust restoration (DRR) framework for the recovery of outage power considering uncertain PV outputs and demands. This framework is presented in two subsequent steps. In the first step, optimal decisions regarding the network configurations are generated. The second step then computes the modified dynamic Distflow equations and constraints under consideration of the worst operating conditions over the associated uncertainty sets with the aim of maximizing the recovery of outage power. The DRR model is formulated as a bi-level mixed-integer linear programming problem. A decomposition algorithm in a master-sub structure is used to solve the resulting system. The results of case studies show that the proposed DRR model yields obvious advantages over the existing deterministic dynamic restoration model in terms of robustness against system uncertainties.
\end{abstract}

Index Terms-Distributed generators, dynamic restoration, power distribution networks, robust optimization.

\section{INTRODUCTION}

$\mathrm{W}^{1}$ ITH the rapid development of the modern industry, the power distribution network is expected to yield much higher reliability and resilience to avoid the customer losses from widespread power outages or blackouts [1]-[5]. Effective fault restoration strategies are expected to play a major role in the self-healing of distribution networks. However, the increasing penetration of photovoltaic (PV) generators, which are volatile and intermittent, introduces new threats to the restoration process [6]-[8]. For instance, the restoration task is time-consuming, and the PV outputs and demands will fluctuate during this process. As for the uncertainty risks, using a conventional deterministic model may result in poor restoration performance, with some restoration strategies even leading to failures because security constraints are violated [9]. In this context, uncertainties bring significant challenges and

Manuscript received July 09, 2019; revised October 12, 2019 and December 07, 2019; accepted December 30, 2019. This work was supported in part by the National Natural Science Foundation of China under Grant 51677025, in part by the State Grid Corporation of China under Grant 52110417000A, in part by the 'Summit of the Six Top Talents' Project of Jiangsu Province under Grant XNY-005, and in part by the Australian Research Council Discovery Project under Grant DP170102303. (Corresponding author: Zaijun Wu.)

J. Xu, Z. Wu, S. Cheng and Q. Hu are with the School of Electrical Engineering, Southeast University, Nanjing, Jiangsu 210096, China (e-mail: andyxu@seu.edu.cn; zjwu@seu.edu.cn; cs0819q@163.com; qh@ieee.org).

$\mathrm{X}$. Yu is with the School of Engineering, RMIT University, Melbourne, VIC 3001, Australia (e-mail: x.yu@rmit.edu.au).

Q. Wu is with the Center for Electric Power and Energy, Department of Electrical Engineering, Technical University of Denmark, Kgs. Lyngby 2800, Denmark (e-mail: qw@elektro.dtu.dk). issues to existing deterministic restoration models at the distribution level, and new solutions are required to ensure the immunity and reliability of restoration strategies.

Several studies have investigated the fault restoration considering the uncertainty of bus power injections. For example, [10]-[12] focus on the recovery of power outages considering system uncertainties, where uncertainty models of demand in the optimal restoration are characterized by fuzzy set theory and maximum membership functions. The fuzzy approach is extended in [13] to determine the optimal restoration scenario that provides a minimal total expected cost. This goal is achieved through the application of fuzzy logic for measuring and managing risk. However, these fuzzy set-based restoration models use a large number of prior fuzzy random variables, and determining these numbers is difficult and impracticable for power operators in most situations [14].

In [15], the authors describe a probabilistic approach based on the two-point estimate method to capture the uncertainty of loads in the optimal restoration model, and apply a novel clonal selection algorithm as the optimization tool. However, the uncertainty of distributed generators (DG) outputs is ignored. In [16], a Markov chain-based operation model is designed to describe the uncertainty of intermittent energy sources, and the load restoration problem is modeled as a chance-constrained stochastic program. A novel extended network for restoration optimization in a distribution network considering probability scenarios of DG outputs is presented in [17], and the specific restoration problem is integrated into a unified spanning-tree problem. A two-stage heuristic is developed to solve the presented load restoration problem. However, such Markov chain- and heuristics-based methodologies are difficult to apply to distribution networks in which the infrastructure and/or operating philosophies are different from those for which they were developed [18].

The authors in [19] present a decentralized multi-agent system (MAS) approach to solve the multi-objective restoration problem considering the uncertainty of DG outputs, where the uncertainty of power injections is forecast by generating scenarios using Monte Carlo simulations (MCS). Nevertheless, MAS-based approaches are network-infrastructure-dependent. In [20], the uncertainties of black-start resources are modeled by discretizing the probability distribution of the forecast errors, and a model predictive control-based optimization strategy for microgrid restoration is explored. A two-stage framework coordinating DG for service restoration is presented in [21]. This aims to enhance the resilience of distribution networks, with the forecast uncertainty of DG output described probabilistically. However, the restoration models presented in [20] and [21] have long runtimes when applied to dynamic and uncertain networks [22]. To make up for this deficiency, a novel risk-limiting load restoration problem has been introduced [23] 
that enables an equivalent transformation of the nonlinear optimization into a mixed-integer linear programming (MILP) problem. Moreover, a Gaussian mixture model is used to describe the probability density functions (PDFs) of DG outputs. Nevertheless, PDF-based solutions for uncertain restoration problems may introduce probabilistic risks to the power system reliability [24].

The aforementioned deficiencies of fuzzy/probability set-based solutions are absent from robust restoration approaches, such as those presented in [9], [25], [26], which aim to satisfy all operational constraints during restoration if the bus power injections vary within bounded uncertainty sets. Two types of static robust restoration models based on information gap decision theory are described in [25] and [26]. These seek the optimal strategies for distribution networks, but use oversimplified descriptions of the uncertainty model. Thus, the authors of [9] present an extended description in which the uncertainty of the bus power injections is described by predefined uncertainty sets. However, the following two major shortcomings still exist: (1) The bounds of DG outputs are designed by human experience; for instance, the authors of [9] assume that the uncertainty sets of DG outputs are predefined as $\left[0.7 S_{\mathrm{DG}}, 1.3 S_{\mathrm{DG}}\right]$, where $S_{\mathrm{DG}}$ is the nominal value of DG outputs. Obviously, the bounds ignore the prediction and modeling of DG outputs, and are potentially nonobjective and unrealistic. (2) Dynamic and three-phase unbalanced distribution networks yield more complicated and practical characteristics, which have been ignored in these robust restoration models.

In light of the above considerations, this paper describes a dynamic robust restoration (DRR) framework for unbalanced distribution networks. The main contributions of this paper are as follows.

1) A three-phase DRR model is proposed for a two-stage robust optimization problem, where the uncertainty of PV outputs and load demands are described as interval numbers. The robustness of the fault restoration strategies derived from the DRR can be guaranteed for all scenarios in the uncertainty sets with acceptable optimization performances. The performance of the DRR framework is validated using numerical tests.

2) In the framework, the interval model for PV outputs uses single-hidden layer feedforward networks (SLFNs) and particle swarm optimization (PSO) instead of human experience. The proposed nonparametric interval approach does not require complicated mathematical models, and yields high prediction accuracy and computational efficiency.

3) The original DRR problem is a mixed-integer quadratic constraint programming (MIQCP) model with a tri-level objective function. To facilitate the solution of the model, the dual-transformation and optimal equidistant piecewise-linear algorithms are introduced, resulting in an MILP problem with a bi-level function.

The remainder of this paper is organized as follows. Section II presents the proposed DRR framework, describing the interval modeling for PV outputs and introducing the mathematical formulation and solution approach of the proposed DRR model. Results of simulation experiments based on two test systems are presented in Section III. Conclusions

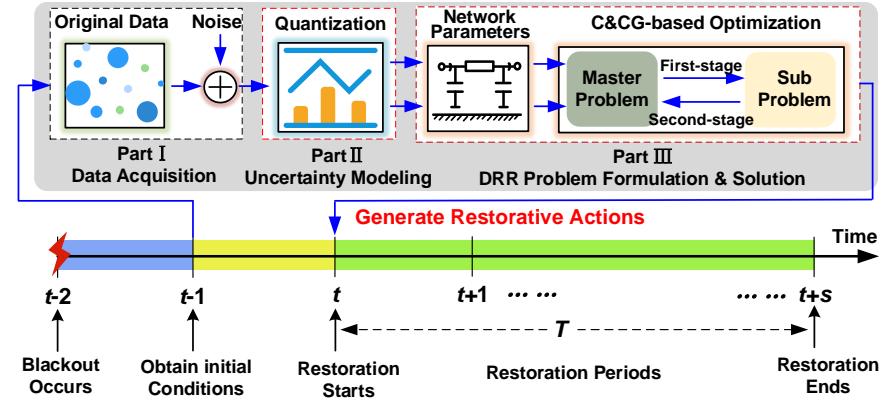

Fig. 1. Look-ahead framework of the proposed DRR.

and a discussion of the obtained results are given in Section IV.

\section{PROPOSED DRR}

After a widespread power outage or blackout in the distribution network, supply restoration aims to recover the power in un-faulted but out-of-service areas. A look-ahead framework of the proposed DRR is depicted in Fig. 1. Initially, distribution system operators (DSOs) identify the potentially affected power consumers and obtain the initial system conditions as original data inputs for the restoration process. The Original Data mainly consist of raw data from day-ahead forecasts of load demands and DG outputs. Each PV is not considered as black-start generation during the restoration process. The Quantization module performs interval modeling for the original data. In this paper, the interval modeling for PV outputs receives special attention. Several MILP constraints with bi-level functions can be incorporated into the Column-and-Constraint Generation (C\&CG)-based Optimization, until the difference between the results of master and sub problems is sufficiently small. Finally, the optimality decisions are generated and provided to the DSO responsible for carrying out restoration actions.

In the following, details about obtaining the bounds of PV outputs are introduced in Subsection II-A, the formulation of the three-phase DRR model is discussed in Subsection II-B, and the solution approach is described Subsection II-C.

\section{A. Interval Modeling for PV Outputs}

In the most robust optimization of distribution networks [9], [25]-[27], the uncertainty modeling of PV outputs lies within bounds that are set according to human experience. However, the given bounds are subjective and may not objectively reflect the fluctuation range of PV outputs. To remedy this deficiency, the proposed DRR framework uses SLFNs and PSO to determine the upper and lower bounds of PV outputs. This approach yields reasonable bounds and be considered as a prerequisite for improving the effectiveness of the DRR solution.

As an alternative tool for quantifying the uncertainty associated with SLFN-based point forecasting, a nonparametric interval forecasting method (IFM) for the PV output bounds has been presented [8]. Nationally, the interval coverage probability (ICP) and interval average width (IAWP) are considered as necessary indices for evaluating the performance of the IFM. These are formulated as

$$
\mathrm{ICP}=\frac{1}{N_{\mathrm{s}}} \sum_{j=1}^{N_{\mathrm{s}}} \epsilon_{j} \times 100 \% \rightarrow P_{a}\left(\boldsymbol{\tau}_{j} \in\left[L_{b}\left(\boldsymbol{t}_{j}\right), U_{b}\left(\boldsymbol{t}_{j}\right)\right]\right)
$$




$$
\text { IAWP }=\frac{1}{N_{\mathrm{s}}} \sum_{j=1}^{N_{\mathrm{s}}}\left(U_{b}\left(\boldsymbol{t}_{j}\right)-L_{b}\left(\boldsymbol{t}_{j}\right)\right) \times 100 \%
$$

where $\epsilon_{j}$ is the coverage behavior of the IFM, $P_{a}(\cdot)$ is the probability, and $L_{b}(\cdot), U_{b}(\cdot)$ are the lower and upper bounds of the forecasting results, respectively. In general, higher values of ICP and lower values of IAWP indicate more accurate and valuable interval forecasting results. Reducing the width of the forecasting intervals often results in a decrease in ICP [28]-[30]. As ICP and IAWP each assess only one aspect of the IFM, a combinational coverage width-based criterion (CWC) is used for simultaneously evaluating the performance of IFM from both perspectives:

$$
\mathrm{CWC}=\operatorname{IAWP}\left(1+\gamma(\mathrm{ICP}) e^{-\vartheta(\mathrm{ICP}-\mu)}\right)
$$

where $\mu$ is the allowable value of the coverage probability and $\vartheta$ is a parameter magnifying the difference between ICP and $\mu$. The hyperparameters $\vartheta$ and $\mu$ control the location and magnitude of CWC. This criterion finds a trade-off between the informativeness (IAWP) and validity (ICP) of the IFM. In terms of optimization theories, CWC is considered a nonlinear and nondifferentiable problem, and a well-known PSO algorithm [31] is employed for its solution. Details of this IFM for PV outputs can be found in [8]. Reasonable bounds of the PV outputs, including the active power output $\left[\underline{P}_{\mathrm{PV}}, \bar{P}_{\mathrm{PV}}\right]$ and the reactive power output $\left[\underline{Q}_{\mathrm{PV}}, \bar{Q}_{\mathrm{PV}}\right]$, are obtained for the DRR, replacing the uncertainty set designed by human experience.

\section{B. Problem Formulation}

\section{1) Original MIQCP-based optimization problem}

Essentially, a restoration strategy is an optimal combination of open and closed switches that achieves the objective within the operating constraints. Unlike existing deterministic dynamic restoration (DDR) models [6], [32], the proposed DRR model characterizes DG outputs and load demands as affine numbers instead of deterministic predictive values. In the proposed DRR model, the interval numbers are converted into affine numbers (see Definition), which are less conservative for robust optimization (RO) than interval values [24].

Definition An affine number $\tilde{a}$ is a set of real numbers. Let AR denote an affine number space. For an affine number $\tilde{a} \in A R$, there is

$$
\tilde{a}=\frac{1}{2}(\underline{a}+\bar{a})+\frac{1}{2}(\bar{a}-\underline{a}) \cdot \varepsilon
$$

where $a$ and $\bar{a}$ are the lower and upper limits of an interval variable $[a]$ and $\varepsilon$ is the uncertain noise in the interval $[-1,1]$. The basic arithmetic operations,,$+- \times$, and $\div$ for affine numbers, as well as logic operations such as $U$ and $\cap$, are described in [24].

In this context, the restoration problem is introduced as a two-stage RO model. The optimal restoration decisions are intended to maximize the recovery of outage power in the first stage, where the switching status represents the decision variables. In the second stage, the worst-case fluctuation scenarios of bus injections (those that jeopardize load active power recovery) are sought from the predefined uncertainty sets. Following verification of the worst-case fluctuation scenario, several optimal decisions are generated to restore as much outage load as possible, and then the final restoration strategy is determined. The proposed two-stage DRR model under consideration of the priority customers is formulated as

$$
\text { obj. } \max _{\left\{\alpha_{\left.i k^{t}, \beta_{i k}^{t}\right\}}\right\} \in \Omega\left\{\varepsilon_{i, \mathrm{~L}}^{\varphi,}, \varepsilon_{i, \mathrm{G}}^{\varphi, t}\right\} \in \Delta} \max \sum_{t=1}^{T} \sum_{\varphi=\mathrm{a}}^{\mathrm{c}} \sum_{j \in \mathrm{V}_{\text {out }}}\left(P_{j, \mathrm{~L}}^{\varphi, t} \zeta_{j} \Delta d_{t}\right)
$$

where $T$ is the number of time periods, $t$ is the index of time periods running from 1 to $T . \varphi \in\{\mathrm{a}, \mathrm{b}, \mathrm{c}\}$ is the phase. $\alpha_{i k}^{t}$ is the binary status variable of branch $i k$ (if branch $i k$ is connected, $\alpha_{i k}^{t}=1$, otherwise, $\left.\alpha_{i k}^{t}=0\right)$ ). $\beta_{i k}$ is the binary variable (if bus $k$ is the parent of bus $i, \beta_{i k}^{t}=1$, otherwise, $\beta_{i k}^{t}=0$ ), and $\boldsymbol{\Omega}$ is the set of feasible network topologies. Here, $i k$ only represents branches with a sectionalizing switch and a tie switch. $\varepsilon_{i, \mathrm{~L}}^{\varphi, t}$ and $\varepsilon_{i, \mathrm{G}}^{\varphi, t}$ are variables used for describing the uncertainty noise of load demands and PV outputs, respectively, and $\boldsymbol{\Delta}$ is the uncertainty set pertaining to the load demands and PV outputs. $\mathbf{V}_{\text {out }}$ is the set of buses in the faulted (outage) area. $P_{j, \mathrm{~L}}^{\varphi, t}$ is the active power demand at bus $j$ during the restoration process. It is assumed that each load can be controlled by a controller that connects/disconnects the load to/from the network, and the recovery of the power outage can be regulated continuously. $\zeta_{j}$ is the weighting factor of importance at bus $j$. $\Delta d_{t}$ is the time horizon of the $t^{\text {th }}$ time period.

During the optimization process, the following constraints should be accurately preserved:

- Modified dynamic Distflow constraint

$$
\begin{aligned}
\widehat{V}_{i}^{\varphi, t}-\widehat{V}_{k}^{\varphi, t} \leq & M_{i k}^{t}+2\left(P_{i k}^{\varphi, t} r_{i k}^{\varphi}+Q_{i k}^{\varphi, t} x_{i k}^{\varphi}\right)- \\
& -\frac{\left(P_{i k}^{\varphi, t}\right)^{2}+\left(Q_{i k}^{\varphi, t}\right)^{2}}{\left(v_{i}^{\varphi, t}\right)^{2}}\left[\left(r_{i k}^{\varphi}\right)^{2}+\left(x_{i k}^{\varphi}\right)^{2}\right] \\
\hat{V}_{i}^{\varphi, t}-\hat{V}_{k}^{\varphi, t} \geq- & M_{i k}^{t}+2\left(P_{i k}^{\varphi, t} r_{i k}^{\varphi}+Q_{i k}^{\varphi, t} x_{i k}^{\varphi}\right)- \\
& -\frac{\left(P_{i k}^{\varphi, t}\right)^{2}+\left(Q_{i k}^{\varphi, t}\right)^{2}}{\left(v_{i}^{\varphi, t}\right)^{2}}\left[\left(r_{i k}^{\varphi}\right)^{2}+\left(x_{i k}^{\varphi}\right)^{2}\right] \\
M_{i k}^{t}= & \left(1-\alpha_{i k}^{t}\right) M,\{i, k\} \in \boldsymbol{\Phi}^{N}
\end{aligned}
$$

where $P_{i k}^{\varphi, t}$ and $Q_{i k}^{\varphi, t}$ are the active and reactive power flows of branch $i k$, respectively, $r_{i k}^{\varphi}$ and $x_{i k}^{\varphi}$ are the known resistance and reactance values of branch $i k$, respectively, $I_{i k}^{\varphi, t}$ is the current flow of branch $i k$, and $\widehat{V}_{i}^{\varphi, t}=\left(V_{i}^{\varphi, t}\right)^{2}$, where $V_{i}^{\varphi, t}$ is the voltage magnitude at bus $i$. Details of the original three-phase Distflow equations can be found in [33]. Here, a big-M method is employed to cancel the constraints in disconnected branches.

- Power balance constraint at buses

$$
\begin{aligned}
& \sum_{i \in \mathbf{m}^{k}}\left[P_{i k}^{\varphi, t}-\frac{\left(P_{i k}^{\varphi, t}\right)^{2}+\left(Q_{i k}^{\varphi, t}\right)^{2}}{\left(v_{i}^{\varphi, t}\right)^{2}} r_{i k}^{\varphi}\right]=\sum_{m \in \mathbf{n}^{k}} P_{k m}^{\varphi, t}+\tilde{P}_{k}^{\varphi, t} \\
& \sum_{i \in \mathbf{m}^{k}}\left[Q_{i k}^{\varphi, t}-\frac{\left(P_{i k}^{\varphi, t}\right)^{2}+\left(Q_{i k}^{\varphi, t}\right)^{2}}{\left(v_{i}^{\varphi, t}\right)^{2}} x_{i k}^{\varphi}\right]=\sum_{m \in \mathbf{n}^{k}} Q_{k m}^{\varphi, t}+\tilde{Q}_{k}^{\varphi, t} \\
& \tilde{P}_{k}^{\varphi, t}=\tilde{P}_{k, \mathrm{G}}^{\varphi, t}-\tilde{P}_{k, \mathrm{~L}}^{\varphi, t}, \quad \tilde{Q}_{k}^{\varphi, t}=\tilde{Q}_{k, \mathrm{G}}^{\varphi, t}-\tilde{Q}_{k, \mathrm{~L}}^{\varphi, t} \\
& \sum_{k \in \Phi^{N}}\left|\varepsilon_{k, \mathrm{G}}^{\varphi, t}\right| \leq \Gamma_{\mathrm{G}} \sqrt{N_{\mathrm{G}}}, \quad \sum_{k \in \boldsymbol{\Phi}^{N}}\left|\varepsilon_{k, \mathrm{~L}}^{\varphi, t}\right| \leq \Gamma_{\mathrm{L}} \sqrt{N_{\mathrm{L}}}
\end{aligned}
$$

where $\mathbf{m}^{k}$ is the set of start nodes in the lines that terminate at bus $k, \mathbf{n}^{k}$ is the set of end buses in the lines that start at bus $k$, $\tilde{P}_{k}^{\varphi, t}$ and $\tilde{Q}_{k}^{\varphi, t}$ are totally active and reactive power injections described in affine form at bus $k$, respectively, $\tilde{P}_{k, \mathrm{G}}^{\varphi, t}$ and $\tilde{Q}_{k, \mathrm{G}}^{\varphi, t}$ are active and reactive power outputs of the PV described in affine form at bus $k$, respectively, $\widetilde{P}_{k, \mathrm{~L}}^{\varphi, t}$ and $\widetilde{Q}_{k, \mathrm{~L}}^{\varphi, t}$ are active and reactive power demands of the load described in affine form at bus $k$, respectively, $\Gamma_{\mathrm{G}} \in[0,1]$ and $\Gamma_{\mathrm{L}} \in[0,1]$ are variables that 
serve as a budget to control the uncertainty of DG outputs and load demands, respectively, and $N_{\mathrm{G}}$ and $N_{\mathrm{L}}$ are the numbers of PVs and loads, respectively. The two auxiliary constraints in (12) are derived from the uncertainty budget approach. These are employed to control the conservativeness of the proposed DRR model.

- Branch capacity constraint

$$
\begin{aligned}
\alpha_{i k}^{t} P_{i k}^{\varphi} & \leq P_{i k}^{\varphi, t} \leq \alpha_{i k}^{t} \bar{P}_{i k}^{\varphi} \\
\alpha_{i k}^{t} \underline{Q}_{i k}^{\varphi} & \leq Q_{i k}^{\varphi, t} \leq \alpha_{i k}^{t} \bar{Q}_{i k}^{\varphi}
\end{aligned}
$$

where $\underline{P}_{i k}^{\varphi}$ and $\bar{P}_{i k}^{\varphi}$ are the minimum and maximum active power flows permitted on branch $i k$, respectively, $\underline{Q}_{i k}^{\varphi}$ and $\bar{Q}_{i k}^{\varphi}$ are the minimum and maximum reactive power flows permitted on branch $i k$, respectively, and $\alpha_{i k}^{t}$ is applied to limit the capacity to zero in disconnected branches.

- Bus voltage security constraint

$$
\underline{V}_{i}^{\varphi} \leq V_{i}^{\varphi, t} \leq \bar{V}_{i}^{\varphi}
$$

where $\underline{V}_{i}^{\varphi}$ and $\bar{V}_{i}^{\varphi}$ are the minimum and the maximum voltage magnitude permitted at bus $i$.

- Radial operation constraint

$$
\begin{gathered}
\beta_{i k}^{t}+\beta_{k i}^{t}=\alpha_{i k}^{t}, \quad i \in \boldsymbol{\Phi}^{N}, \quad k \in \boldsymbol{\Phi}^{i} \\
\sum_{k \in \boldsymbol{\Phi}^{i}} \beta_{i k}^{t}=1, \beta_{1 k}^{t}=0, \quad i \in \boldsymbol{\Phi}^{N-1}, k \in \boldsymbol{\Phi}^{1}
\end{gathered}
$$

where $\boldsymbol{\Phi}^{N}$ is the set of buses in the distribution network with size $N, \boldsymbol{\Phi}^{i}$ is the set of buses connected to bus $i, \boldsymbol{\Phi}^{N-1}$ is the set of buses without the slack bus, and $\boldsymbol{\Phi}^{1}$ is the set of buses connected to the slack bus. Equations (16) and (17) ensure there are no loops in the network.

At this point, the DRR model is complicated and its solution is challenging because of the tri-level objective function and quadratic mixed-integer constraints. Fortunately, the inner maximization model of the objective function (5) can be replaced by its Karush-Kuhn-Tucker (KKT) conditions, which are necessary and sufficient conditions of optimality for a strictly linear program. Consequently, the original tri-level objective function can be converted into a "max-min" optimization by adding the dual problem constraints and their corresponding complementarity slackness conditions [34]. Details of the new objective function are given at the end of this subsection.

\section{2) Linearization approach for quadratic terms}

The nonlinear expression of the branch loss in the constraints, $\left[\left(P_{i k}^{\varphi, t}\right)^{2}+\left(Q_{i k}^{\varphi, t}\right)^{2}\right] /\left(V_{i}^{\varphi, t}\right)^{2}$, can be approximated by $\left(P_{i k}^{\varphi, t}\right)^{2}+$ $\left(Q_{i k}^{\varphi, t}\right)^{2}$, assuming that the bus voltage $V_{i}^{\varphi, t} \approx 1.0 \mathrm{pu}$ [34]. Furthermore, $\left(P_{i k}^{\varphi, t}\right)^{2}$ and $\left(Q_{i k}^{\varphi, t}\right)^{2}$ can be accurately approximated into piecewise-linear terms using the optimal equidistant piecewise-linear algorithm [35]. In doing so, consider the function $h\left(P_{i k}^{\varphi, t}\right)=\left(P_{i k}^{\varphi, t}\right)^{2}$ and let the maximum linear approximation error be $\mathrm{C}$. As shown in Fig. 2, the $(s+1)^{\text {th }}$ linear segment, $I_{i k, s+1}^{\varphi, t}$, can be obtained by

$$
\begin{gathered}
h\left(P_{i k, s s}^{\varphi, t}\right)-h\left(P_{i, s}^{\varphi, t}\right)+2 \mathrm{C}=h^{\prime}\left(P_{i, s, s}^{\varphi, t}\right)\left(P_{i k, s s}^{\varphi, t}-P_{i k, s}^{\varphi, t}\right) \\
h\left(P_{i k, s+1}^{\varphi, t}\right)-h\left(P_{i k, s s}^{\varphi, t}\right)-2 \mathrm{C}=h^{\prime}\left(P_{i k, s S}^{\varphi, t}\right)\left(P_{i k, s+1}^{\varphi, t}-P_{i k, s s}^{\varphi, t}\right)
\end{gathered}
$$

where $s \in \boldsymbol{\Phi}^{S}$, $\boldsymbol{\Phi}^{S}$ is the set of segments with size $S$. $P_{i k, s s}^{\varphi, t}$ is the point-cut (see Fig. 2), and $h^{\prime}\left(P_{i k, S S}^{\varphi, t}\right)$ is the first-order derivative of $h\left(P_{i k, s s}^{\varphi, t}\right)$. Once $P_{i k, 1}^{\varphi, t}$ (the starting point of the first

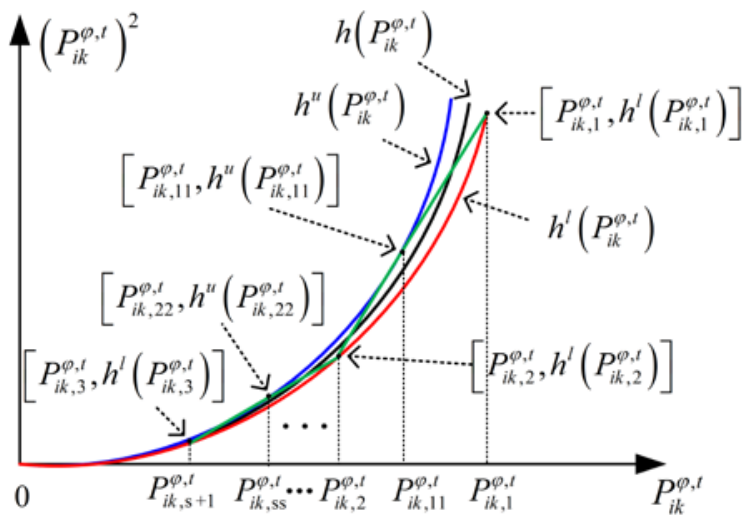

Fig. 2. Schematic diagram of finding the minimum segment length, where the black line represents the function $h\left(P_{i k}^{\varphi, t}\right)$, the red line represents the function $h^{l}\left(P_{i k}^{\varphi, t}\right)=h\left(P_{i k}^{\varphi, t}\right)-\mathrm{C}$, the blue line represents the function $h^{u}\left(P_{i k}^{\varphi, t}\right)=$ $h\left(P_{i k}^{\varphi, t}\right)+\mathrm{C}$, and the green line represents the tangent of the function $h^{u}\left(P_{i k}^{\varphi, t}\right)$.

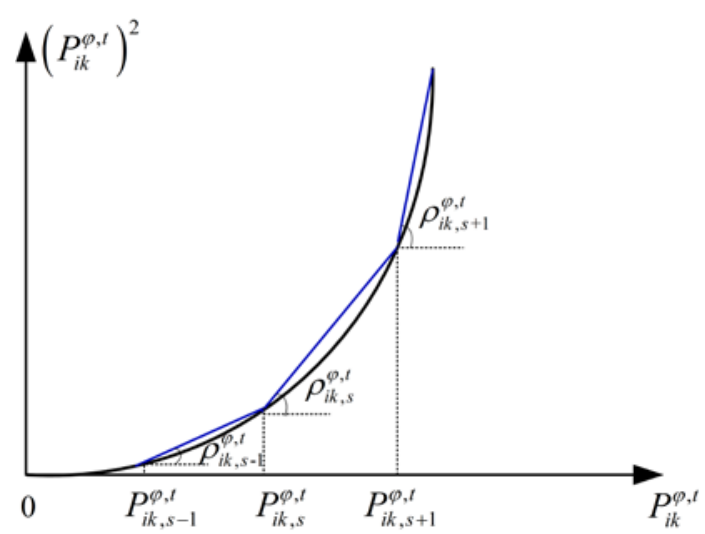

Fig. 3. Piecewise-linear approximation of the $\left(P_{i k}^{\varphi, t}\right)^{2}$.

segment) has been set, all $P_{i k, s}^{\varphi, t}$ can be obtained using (18)-(19). Subsequently, the minimum segment length, $l=P_{i k, s+1}^{\varphi, t}-P_{i k, s}^{\varphi, t}$, is achieved. Consequently, the active power flow $P_{i k}^{\varphi, t}$ is divided into $S$ equal-length segments. Further details of the basic optimal equidistant piecewise-linear algorithm can be found in [35].

The original quadratic term $\left(P_{i k}^{\varphi, t}\right)^{2}$ in the constraints can be rewritten as follows (see Fig. 3):

$$
\left(P_{i k}^{\varphi, t}\right)^{2} \approx \sum_{s \in \Phi} \rho_{i k, s}^{\varphi, t} P_{i k, s}^{\varphi, t}
$$

where $\boldsymbol{\Phi}^{S}$ is the set of segments with size $S$ and $\rho_{i k, S}^{\varphi, t}$ is the slope of the $s^{\text {th }}$ linear segment of $P_{i k}^{\varphi, t}$.

Similarly, $\left(Q_{i k}^{\varphi, t}\right)^{2}$ in the constraints can be rewritten as

$$
\left(Q_{i k}^{\varphi, t}\right)^{2} \approx \sum_{s \in \boldsymbol{\Phi}} s \gamma_{i k, s}^{\varphi, t} Q_{i k, s}^{\varphi, t}
$$

where $\gamma_{i k, s}^{\varphi, t}$ is the slope of the $s^{\text {th }}$ linear segment of $Q_{i k}^{\varphi, t}$.

As both $P_{i k, s}^{\varphi, t}$ and $Q_{i k, s}^{\varphi, t}$ are nonnegative, several nonnegative variables $P_{i k,+}^{\varphi, t}, P_{i k,-}^{\varphi, t}, Q_{i k,+}^{\varphi, t}$, and $Q_{i k,-}^{\varphi, t}$ are introduced to account for the reverse negative power flows. Consequently, the following constraints of (20) and (21) are derived:

$$
\begin{gathered}
P_{i k}^{\varphi, t}=P_{i k,+}^{\varphi, t}-P_{i k,-}^{\varphi, t}, P_{i k,+}^{\varphi, t} \geq 0, P_{i k,-}^{\varphi, t} \geq 0 \\
\left|P_{i k}^{\varphi, t}\right|^{\varphi, t} P_{i k,+}^{\varphi, t}+P_{i k,-}^{\varphi, t}=\sum_{s \in \Phi^{S}} P_{i k, s}^{\varphi, t}, 0 \leq P_{i k, s}^{\varphi, t} \leq \bar{P}_{i k, s}^{\varphi, t} \\
Q_{i k}^{\varphi, t}=Q_{i k,+}^{\varphi, t}-Q_{i k,-}^{\varphi, t}, Q_{i k,+}^{\varphi, t} \geq 0, Q_{i k,-}^{\varphi, t} \geq 0
\end{gathered}
$$




$$
\left|Q_{i k}^{\varphi, t}\right|=Q_{i k,+}^{\varphi, t}+Q_{i k,-}^{\varphi, t}=\sum_{s \in \Phi^{S}} Q_{i k, s}^{\varphi, t}, \quad 0 \leq Q_{i k, s}^{\varphi, t} \leq \bar{Q}_{i k, s}^{\varphi, t}
$$

where $\bar{P}_{i k, s}^{\varphi, t}$ and $\bar{Q}_{i k, s}^{\varphi, t}$ denote the upper limits of $P_{i k, s}^{\varphi, t}$ and $Q_{i k, s}^{\varphi, t}$.

Hence, (6)-(7) and (9)-(10) can be rewritten as follows, where $R=\left[\left(r_{i k}^{\varphi}\right)^{2}+\left(x_{i k}^{\varphi}\right)^{2}\right]$ :

$$
\begin{aligned}
& \widehat{V}_{i}^{\varphi, t}-\widehat{V}_{k}^{\varphi, t} \leq M_{i k}^{t}+2\left(P_{i k}^{\varphi, t} r_{i k}^{\varphi}+Q_{i k}^{\varphi, t} x_{i k}^{\varphi}\right)- \\
& -R\left(\sum_{s \in \boldsymbol{\Phi}^{s}} \rho_{i k, s}^{\varphi, t} P_{i k, s}^{\varphi, t}+\sum_{s \in \boldsymbol{\Phi}^{s}} \gamma_{i k, s}^{\varphi, t} Q_{i k, s}^{\varphi, t}\right) \\
& \widehat{V}_{i}^{\varphi, t}-\widehat{V}_{k}^{\varphi, t} \geq-M_{i k}^{t}+2\left(P_{i k}^{\varphi, t} r_{i k}^{\varphi}+Q_{i k}^{\varphi, t} x_{i k}^{\varphi}\right)- \\
& -R\left(\sum_{s \in \boldsymbol{\Phi} s} \rho_{i k, s}^{\varphi, t} P_{i k, s}^{\varphi, t}+\sum_{s \in \boldsymbol{\Phi}^{S}} \gamma_{i k, S}^{\varphi, t} Q_{i k, s}^{\varphi, t}\right) \\
& \sum_{i \in \mathbf{m}^{k}}\left[P_{i k}^{\varphi, t}-r_{i k}^{\varphi}\left(\sum_{s \in \boldsymbol{\Phi}^{S}} \rho_{i k, s}^{\varphi, t} P_{i k, s}^{\varphi, t}+\right.\right. \\
& \left.\left.+\sum_{s \in \boldsymbol{\Phi}^{S}} \gamma_{i k, s}^{\varphi, t} Q_{i k, s}^{\varphi, t}\right)\right]=\sum_{m \in \mathbf{n}^{k}} P_{k m}^{\varphi, t}+\tilde{P}_{k}^{\varphi, t} \\
& \sum_{i \in \mathbf{m}^{k}}\left[Q_{i k}^{\varphi, t}-x_{i k}^{\varphi}\left(\sum_{s \in \boldsymbol{\Phi}} s \rho_{i k, s}^{\varphi, t} P_{i k, s}^{\varphi, t}+\right.\right. \\
& \left.\left.+\sum_{s \in \boldsymbol{\Phi}^{S}} \gamma_{i k, s}^{\varphi, t} Q_{i k, s}^{\varphi, t}\right)\right]=\sum_{m \in \mathbf{n}^{k}} Q_{k m}^{\varphi, t}+\tilde{Q}_{k}^{\varphi, t}
\end{aligned}
$$

3) Resulting MILP-based optimization problem

At this point, the original tri-level MIQCP-based optimization problem of the proposed DRR model can be reformulated as the following bi-level one by adding its $K K T$ conditions:

$$
\begin{aligned}
& \text { obj. } \max _{\left\{\alpha_{i k}^{t}, \beta_{i k}^{t}\right\} \in \Omega\left\{\left\{\varepsilon_{i, \mathrm{~L}}, \varepsilon_{i, \mathrm{G}}^{\varphi, t}\right\} \in \mathbf{\Delta}\right.} \sum_{t=1}^{T} \sum_{\varphi=\mathrm{a}}^{\mathrm{c}} \sum_{j \in \mathrm{V}_{\text {out }}}\left(P_{j, \mathrm{~L}}^{\varphi, t} \zeta_{j} \Delta d_{t}\right) \\
& \text { subject to } \\
& \text { [Constraints (8), (11) - (17), (22) - (29)] } \\
& \iota_{P, i k}^{\varphi, t}-\left(\bar{\lambda}_{P, i k}^{\varphi, t}-\underline{\lambda}_{P, i k}^{\varphi, t}\right)-\left(2 r_{i k}^{\varphi} L_{V, i}^{\varphi, t}+2 r_{i k}^{\varphi} L_{V, k}^{\varphi, t}\right)=0 \\
& \iota_{Q, i k}^{\varphi, t}-\left(\bar{\lambda}_{Q, i k}^{\varphi, t}-\underline{\lambda}_{Q, i k}^{\varphi, t}\right)-\left(2 x_{i k}^{\varphi} L_{V, i}^{\varphi, t}+2 x_{i k}^{\varphi} L_{V, k}^{\varphi, t}\right)=0 \\
& \bar{K}_{V, i}^{\varphi, t}-\underline{K}_{V, i}^{\varphi, t}+L_{V, i}^{\varphi, t}-L_{V, k}^{\varphi, t}=0 \\
& \left\{\bar{\lambda}_{P, i k}^{\varphi, t}, \underline{\lambda}_{P, i k}^{\varphi, t}, \bar{\lambda}_{Q, i k}^{\varphi, t}, \underline{\lambda}_{Q, i k}^{\varphi, t}, \bar{K}_{V, i}^{\varphi, t}, \underline{K}_{V, i}^{\varphi, t}, L_{V, i}^{\varphi, t}, L_{V, k}^{\varphi, t}\right\} \geq 0 \\
& L_{V, i}^{\varphi, t}\left[\begin{array}{c}
\hat{V}_{i}^{\varphi, t}-\widehat{V}_{k}^{\varphi, t}-M_{i k}^{t}-2\left(P_{i k}^{\varphi, t} r_{i k}^{\varphi}+Q_{i k}^{\varphi, t} x_{i k}^{\varphi}\right)+ \\
+R\left(\sum_{s \in \boldsymbol{\Phi}} s \rho_{i k, s}^{\varphi, t} P_{i k, s}^{\varphi, t}+\sum_{s \in \boldsymbol{\Phi}} s \gamma_{i k, S}^{\varphi, t} Q_{i k, s}^{\varphi, t}\right)
\end{array}\right]=0 \\
& L_{V, i}^{\varphi, t}\left[\begin{array}{c}
\widehat{V}_{k}^{\varphi, t}-\widehat{V}_{i}^{\varphi, t}-M_{i k}^{t}+2\left(P_{i k}^{\varphi, t} r_{i k}^{\varphi}+Q_{i k}^{\varphi, t} x_{i k}^{\varphi}\right)+ \\
+R\left(\sum_{s \in \Phi^{S}} \rho_{i k, s}^{\varphi, t} P_{i k, s}^{\varphi, t}+\sum_{s \in \boldsymbol{\Phi}} S \gamma_{i k, S}^{\varphi, t} Q_{i k, s}^{\varphi, t}\right)
\end{array}\right]=0 \\
& \underline{\lambda}_{P, i k}^{\varphi, t}\left(-\alpha_{i k}^{t} \underline{P}_{i k}^{\varphi}-P_{i k}^{\varphi, t}\right)=0, \bar{\lambda}_{P, i k}^{\varphi, t}\left(P_{i k}^{\varphi, t}-\alpha_{i k}^{t} \bar{P}_{i k}^{\varphi}\right)=0 \\
& \underline{\lambda}_{Q, i k}^{\varphi, t}\left(-\alpha_{i k}^{t} \underline{Q}_{i k}^{\varphi}-Q_{i k}^{\varphi, t}\right)=0, \bar{\lambda}_{Q, i k}^{\varphi, t}\left(Q_{i k}^{\varphi, t}-\alpha_{i k}^{t} \bar{Q}_{i k}^{\varphi}\right)=0 \\
& \underline{K}_{V, i}^{\varphi, t}\left(\underline{V}_{i}^{\varphi}-V_{i}^{\varphi, t}\right)=0, \bar{K}_{V, i}^{\varphi, t}\left(V_{i}^{\varphi, t}-\bar{V}_{i}^{\varphi}\right)=0
\end{aligned}
$$

where $\iota_{P, i k}^{\varphi, t}, \iota_{Q, i}^{\varphi, t} L_{V, i}^{\varphi, t}$, and $L_{V, k}^{\varphi, t}$ are dual variables associated with the modified dynamic Distflow constraint. $\bar{\lambda}_{P, i k}^{\varphi, t}, \underline{\lambda}_{P, i k}^{\varphi, t}$, $\bar{\lambda}_{Q, i k}^{\varphi, t}$, and $\underline{\lambda}_{Q, i k}^{\varphi, t}$ are dual variables associated with the branch capacity constraint. $\bar{K}_{V, i}^{\varphi, t}$ and $\underline{K}_{V, i}^{\varphi, t}$ are dual variables associated with the bus voltage security constraint. Constraints (36)-(40) are the complementarity slackness conditions.

\section{Problem Solution Strategy}

The proposed DRR model is cast as a two-stage RO problem, which can be solved using the C\&CG algorithm [9], [24]. The C\&CG algorithm is implemented in a master-sub form. The inner C\&CG is used for solving the sub problem, whereby the worst-case fluctuation scenarios of bus injections are sought for a given first-stage decision variable (the switching status) that provides an upper bound. Several new variables and constraints are added to the outer C\&CG algorithm to solve the master
Algorithm C\&CG for solving the DRR problem

Step 1: Initialization

- Set the lower bound $L_{b}$ and the upper bound $U_{b}$ of the objective function (32) as: $U_{b}=+\infty$ and $L_{b}=0$.

- Set the convergence tolerance $\epsilon=10^{-5}$, and the iteration index $\tau^{\prime}=1$.

Step 2: Outer C\&CG: Master Problem Solution

- Select a feasible scenario of DG outputs and load demands $\left\{\varepsilon_{i, \mathrm{~L}}^{(*) \varphi, t}, \varepsilon_{i, \mathrm{G}}^{(*) \varphi, t}\right\} \in \Delta$. For $\tau=1, \cdots, \tau^{\prime}-1$, solve the following master problem

obj. $F_{\mathrm{M}}=\max _{\left\{\alpha_{i k}^{*}, \beta_{i k}^{*}\right\} \in \Omega} \eta$

\section{subject to}

$$
\eta \leq \sum_{t=1}^{T} \sum_{\varphi=\mathrm{a}}^{\mathrm{c}} \sum_{i k=1}^{N_{b}} \sum_{j \in \mathrm{V}_{\text {out }}} P_{j, \mathrm{~L}(\tau)}^{\varphi, t} \zeta_{j} \Delta d_{t}
$$

$P_{k(\tau)}^{\varphi, t}=P_{k, \mathrm{G}(\tau)}^{\varphi, t}-P_{k, \mathrm{~L}(\tau)}^{\varphi, t}, Q_{k(\tau)}^{\varphi, t}=Q_{k, \mathrm{G}(\tau)}^{\varphi, t}-Q_{k, \mathrm{~L}(\tau)}^{\varphi, t}$

$P_{i k(\tau)}^{\varphi, t}=P_{i k,+(\tau)}^{\varphi, t}-P_{i k,-(\tau)}^{\varphi, t}, P_{i k,+(\tau)}^{\varphi, t} \geq 0, P_{i k,-(\tau)}^{\varphi, t} \geq 0$

[Constraints (8), (12) - (17), (22) - (29)]

- Obtain the optimal solution of the master problem

$$
\left\{\alpha_{i k}^{(*)}, \beta_{i k}^{(*)}\right\} \text {, and } F_{\mathrm{M}} \text {. }
$$

- Update the lower bound by $L_{b}=\max \left\{L_{b}, F_{\mathrm{M}}\right\}$.

Step 3: Inner C\&CG: Sub Problem Solution

- Assume that the switching status $\left\{\alpha_{i k}, \beta_{i k}\right\}$ is fixed to the solution produced from the master problem in Step 2). Then solve the following sub problem

$$
\text { obj. } F_{\mathrm{S}}=\min _{\left\{\varepsilon_{i, \mathrm{~L}}^{(*), t}, \varepsilon_{i, \mathrm{G}}^{(*) \varphi, t}\right\} \in \Delta} \sum_{j \in V_{\text {out }}} P_{j, \mathrm{~L}}^{\varphi, t} \zeta_{j} \Delta d_{t}
$$

\section{subject to}

[Constraints (8), (11) - (15), (22) - (29), (32) - (40)]

- Obtain the worst-case fluctuation scenario of bus power injections $\left\{P_{k}^{(*) \varphi, t}, Q_{k}^{(*) \varphi, t}\right\}$, and $F_{\mathrm{S}}$.

- Update the upper bound by $U_{b}=\min \left\{U_{b}, F_{\mathrm{S}}\right\}$.

- Set $P_{k}^{(*) \varphi, t}=P_{k(\tau)}^{\varphi, t}$ and $Q_{k}^{(*) \varphi, t}=Q_{k(\tau)}^{\varphi, t}$.

Step 4: Convergence Check

- If $\left|U_{b}-L_{b}\right| \leq \epsilon$, terminate;

- Else, update $\tau=\tau+1$, and go back to Step 2).

problem, with the objective of obtaining a lower bound. The sub and master problems are solved iteratively until the interval between the upper and lower bounds is less than a preset convergence tolerance.

The master-sub iterative process for solving the proposed DRR problem is described in the Algorithm. The optimization problems involved in both inner and outer C\&CG algorithms are MILP models, which can be efficiently handled using off-the-shelf commercial solvers.

\section{CASE STUdiES AND RESUlTS}

The proposed DRR approach is formulated in the MATLAB environment, with the GUROBI package employed for MILP computations. This section presents the results and comparisons of numerical simulation tests performed on a personal computer with an Intel (R) Core (TM) i5-2400 CPU @ $3.10 \mathrm{GHz}$ and $4 \mathrm{~GB}$ main memory. 


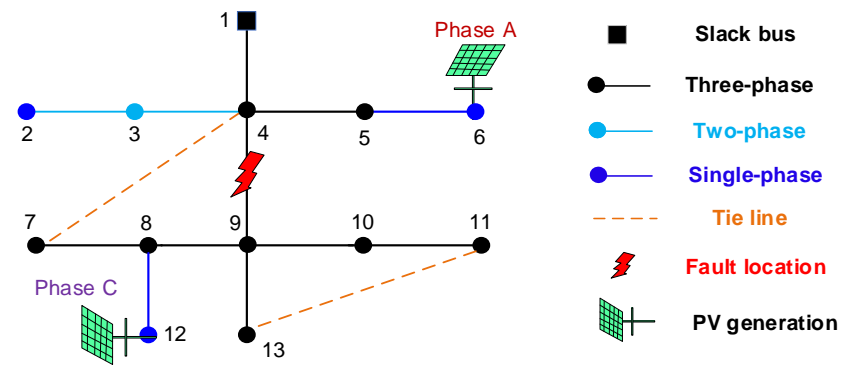

Fig. 4. One-line of the modified IEEE 13-bus network.

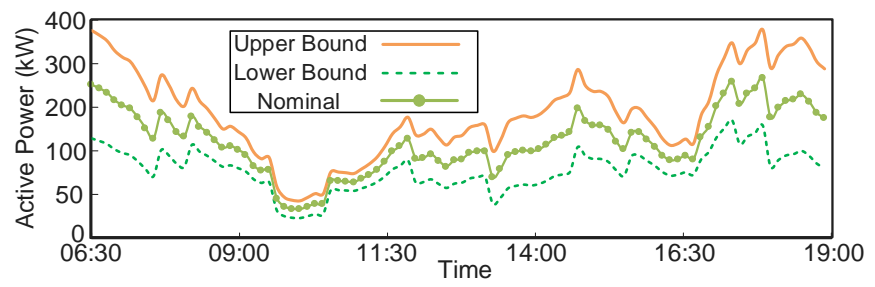

Fig. 5. Daily interval profiles of load power demands (phase C) at bus 11.

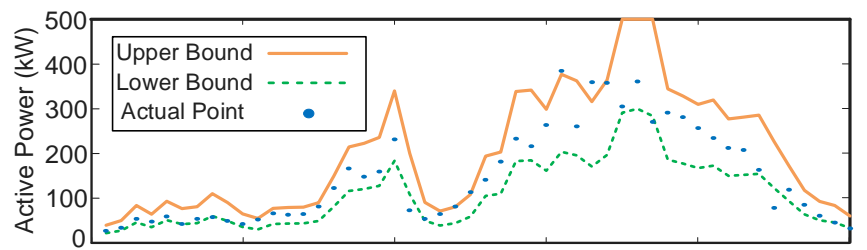

(a)

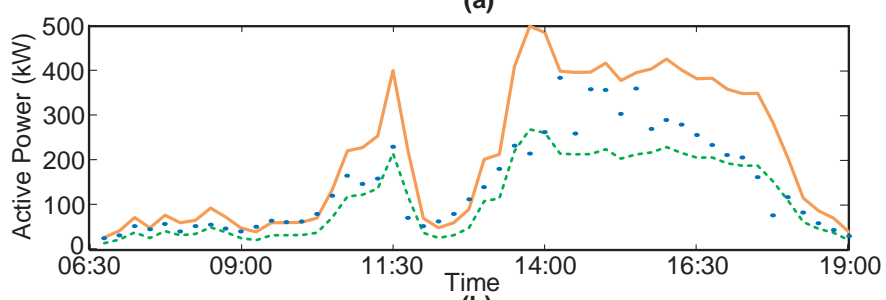

(b)

Fig. 6. Daily interval profiles of PV outputs obtained by two methods: (a) results obtained by the proposed method, (b) results obtained by the existing method.

TABLE I

COMPARISONS OF FORECASTING INTERVALS OBTAINED BY TWO METHODS

\begin{tabular}{ccccc}
\hline Prediction Method & ICP(\%) & INAW(\%) & CWC & CPU Time (s) \\
\hline Existing Method & 86.049 & 62.183 & 0.096 & 2.017 \\
Proposed Method & 92.381 & 59.916 & 0.171 & 0.782 \\
\hline \hline
\end{tabular}

\section{A. Small Test System: Modified IEEE 13-Bus Network}

In this test, the modified IEEE 13-bus network is used to illustrate the effectiveness of the proposed DRR framework. This network operates at a nominal voltage of $4.16 \mathrm{kV}$, and the minimum and maximum bus voltage magnitudes are $0.95 \mathrm{pu}$ and $1.05 \mathrm{pu}$. As the original IEEE 13-bus network [36] does not include PV, it is modified through the addition of two single PV generators with an installed capacity of $515.8 \mathrm{~kW}$. The PV generation is assumed to be controlled in the PQ-strategy, and the power factor is set to 0.85 . Loads are categorized as industrial (i.e., bus 2 and bus 7), commercial (i.e., bus 4, bus 8 , and bus 9), and residential (the remaining buses). The total load demands are $1158+j 606 \mathrm{kVA}$ in phase $\mathrm{A}, 973+\mathrm{j} 627 \mathrm{kVA}$ in phase $\mathrm{B}$, and $1137+\mathrm{j} 753 \mathrm{kVA}$ in phase $\mathrm{C}$. This network has six sectionalizing switches (normally closed) and two tie switches (normally open). Its layout is illustrated in Fig. 4. Detailed information (e.g., type, parameters, and capacity limits) of each branch and the locations of all switches can be found in [5]. Moreover, the allowed convergence tolerance of the C\&CG algorithm is set to $10^{-5}$.

In the worst-case fluctuation scenario, the uncertainty sets of load demands and PV outputs are constructed as follows.

1) The nominal daily load profiles are generated in the IEEE-RTS. In each hour, the three-phase load fluctuations at each bus are assumed to deviate by up to $30 \%$ from their nominal values, that is, $\left[\underline{P}_{\mathrm{L}}, \bar{P}_{\mathrm{L}}\right]=\left[0.7 P_{\mathrm{L}}, 1.3 P_{\mathrm{L}}\right],\left[\underline{Q}_{\mathrm{L}}, \bar{Q}_{\mathrm{L}}\right]=$ $\left[0.7 Q_{\mathrm{L}}, 1.3 Q_{\mathrm{L}}\right]$, where $P_{\mathrm{L}}$ and $Q_{\mathrm{L}}$ denote the nominal active and reactive values of each load, respectively. For instance, the partial curve of load fluctuation (active power demand on phase C) at bus 11 is illustrated in Fig. 5 .

2) The historical statistical output of one PV generator and its meteorological information [37] are selected as the IFM inputs introduced in Subsection II-B. The dataset from a summer month (July 2013) consists of data recorded at 15-min intervals each day (from 06:30 to 19:00). The historical statistics of the $\mathrm{PV}$ outputs and corresponding meteorological data of the first 30 days are used as training data. The forecasting bounds of PV outputs for the last day of the month are then obtained using the corresponding meteorological data. The parameters used in the SLFNs training and PSO process are given in [8]. Note that different weather conditions can lead to different uncertainty patterns of PV outputs, thus affecting the decision-making strategies of the DRR models. Qualitatively, larger interval widths for the inputs will produce more conservative robust results [8]. For the sake of convenience, it is assumed that all network buses in the following two cases are subject to the same weather conditions, which means all eight PV generators have the same uncertainty patterns. To show the performance of the IFM proposed in this paper, an existing PDF-based interval prediction method [29] is selected to obtain the daily forecasted bounds of PV outputs using the same datasets. Fig. 6 displays the daily forecasted bounds and true values of PV outputs on July 31, 2013, and the evaluation results of each daily forecasting interval obtained by the two methods are listed in Table I. From these comparisons, it is apparent that the bounds forecast by the proposed method cover a greater percentage of the actual values of PV outputs (a higher value of ICP) and have a higher CWC value than those given by the existing method. The proposed method is also faster than the existing method in obtaining the daily forecasting intervals of PV outputs. This is because the existing PDF-based interval prediction method requires numerous MCS to produce forecasts, which increases its time complexity. Therefore, the proposed IFM yields more accurate bounds and faster computational performance, and should thus be considered as a credible data source for the industrial application of the proposed DRR.

The affine forms of each load demand and PV output can then be obtained using the daily interval power profiles. The initial budget values of load demands $\Gamma_{\mathrm{L}}$ and PV outputs $\Gamma_{\mathrm{G}}$ are set to 1 . In the case of a three-phase fault occurring in branch 49 from 13:00-15:00 (i.e., $T=2$ and $\Delta d_{t}=1$ ), a power outage will occur after isolating the fault. In this context, service 
TABLE II

RESTORATION RESUltS FOR TWO RESTORASTION MODELS UNDER THE SMALL TEST SySTEM

\begin{tabular}{cccccc}
\hline \hline \multirow{2}{*}{ Time Period } & \multirow{2}{*}{ Power Outage $(\mathrm{kW})$} & \multirow{2}{*}{ Model } & \multirow{2}{*}{ Open Line } & \multicolumn{2}{c}{ Total Three-phase Restored Power (kW) } \\
\cline { 4 - 6 } & & & Unfluctuating & Worst-case Fluctuation \\
\hline \multirow{2}{*}{ 13:00 14:00 } & \multirow{2}{*}{795} & DDR & $4-9,9-10$ & 784 & 771 \\
& \multirow{2}{*}{ 14:00 15:00 } & DRR & $4-9,11-13$ & 779 & 779 \\
& \multirow{2}{*}{706} & DDR & $4-9,9-10$ & 698 & 687 \\
\hline \hline
\end{tabular}

TABLE III

RESTORATION RESULTS OF TWO UNCERTAINTY BUDGET VALUES

\begin{tabular}{ccc}
\hline \hline Budget Value & Open Line & $\begin{array}{c}\text { Total Resorted Power } \\
(\mathrm{kW})\end{array}$ \\
\hline$\Gamma_{\mathrm{L}}=0.2, \Gamma_{\mathrm{G}}=0.2$ & $4-9,10-11$ & 784 \\
$\Gamma_{\mathrm{L}}=0.8, \Gamma_{\mathrm{G}}=0.8$ & $4-9,11-13$ & 780 \\
\hline \hline
\end{tabular}

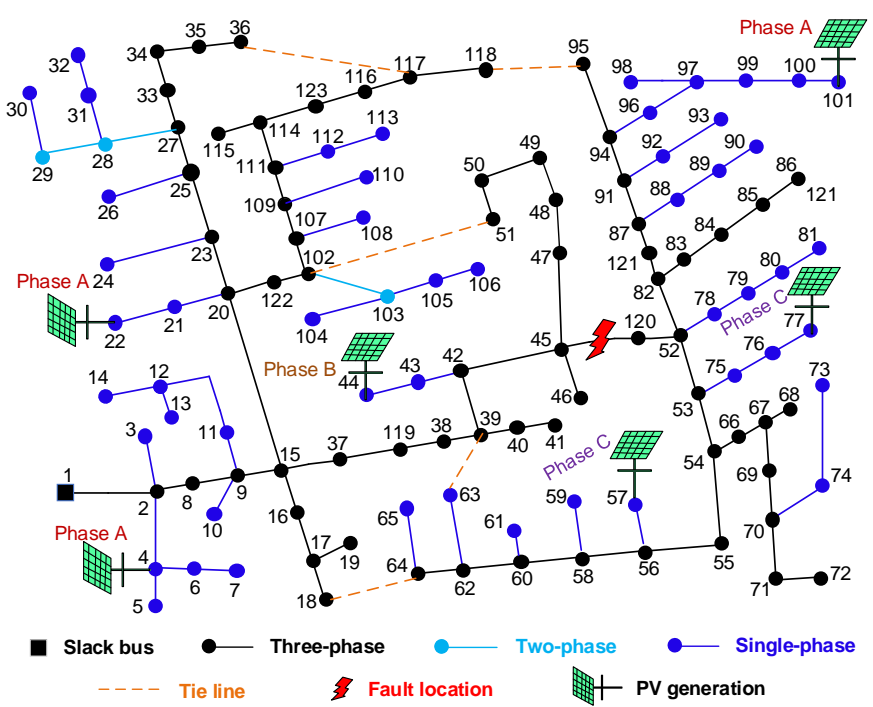

Fig. 7. One-line diagram of the modified IEEE 123-bus test system.

TABLE IV

POWER DEMANDS CHARACTERISTICS OF DIFFERENT LOAD BUSES

\begin{tabular}{ccc}
\multicolumn{2}{c}{ LOWER DEMANDS CHARACTERISTICS OF DIFFERENT LOAD BUSES } \\
\hline Type & Load Bus & Weight \\
\hline Industrial & $\begin{array}{c}3,4,9,10,12,24,26,43,57,63,92, \\
104,113\end{array}$ & 10 \\
Commercial & $\begin{array}{c}5,11,16,44,50,59,61,75,88,96, \\
103,105,108,112,116,118 \\
\text { Remaining Buses }\end{array}$ & 5 \\
Residential & & 1 \\
\hline \hline
\end{tabular}

restoration is conducted using the proposed DRR model and the DDR model [32]. The load demands and PV outputs are nominal in the dynamic deterministic restoration (DDR) model. Consider the nominal active and reactive power outputs of each $\mathrm{PV}$ to be $P_{\mathrm{PV}}=\frac{1}{2}\left(\underline{P}_{\mathrm{PV}}+\bar{P}_{\mathrm{PV}}\right)$ and $Q_{\mathrm{PV}}=\frac{1}{2}\left(\underline{Q}_{\mathrm{PV}}+\bar{Q}_{\mathrm{PV}}\right)$, respectively. The simulation results and comparisons between these two models are now presented.

Table II compares the recovery of outage power under the unfluctuating and worst-case fluctuation scenarios. Here, the worst-case fluctuation scenario for the DDR model refers to the case in which the load demands and PV outputs in each hour remain at their upper bound values during each restoration period. Note that the difference between the optimal topologies and power recoveries are generated using these two restoration models. In the case of the unfluctuating condition, the power recovery of the proposed DRR model in each restoration period is slightly lower than that of the conventional DDR model, because DRR reduces the optimality slightly to immunize the system against any undesired uncertainties of bus power injections. However, under the worst-case fluctuation scenario, the DRR strategies in each restoration period are outperformed by the DDR model, which can recover much higher power outages.

Using the uncertainty budget given in (12), $\Gamma_{\mathrm{G}}$ and $\Gamma_{\mathrm{L}}$ can be tuned to adjust the conservativeness of DRR. Under the worst-case fluctuation scenario in the overall uncertainty sets, DRR attempts restoration with varying budget values. During the first restoration period, datasets of $\Gamma_{\mathrm{G}}$ and $\Gamma_{\mathrm{L}}$ are created to evaluate the impact of the uncertainty budget. The restoration results of those three cases are summarized in Table III. From Table III, it is clear that, under the same worst-case scenarios, the power restored by DRR increases as the budget values $\Gamma_{G}$ and $\Gamma_{\mathrm{L}}$ increase. This is consistent with the intuitive result that a larger budget should yield a more robust strategy. However, the larger budget values could also introduce more conservatism into the restoration results. In reality, a trade-off between the robustness and conservativeness of the proposed DRR model can be reached by changing the budget values of the uncertain power injections.

\section{B. Large Test System: Modified IEEE 123-Bus Network}

This test considers the large-scale modified IEEE 123-bus network to illustrate the effectiveness of the proposed DRR framework. The one-line diagram of the network is shown in Fig. 7. Six single-phase PV generators with an installed capacity of $515.8 \mathrm{~kW}$ are added to the original IEEE 123-bus system. All PV generations are assumed to be controlled using a PQ-strategy, and the power factor is set to 0.85 . This test system operates at a nominal voltage of $4.16 \mathrm{kV}$, the total loads are $1420+\mathrm{j} 775 \mathrm{kVA}$ in phase $\mathrm{A}, 915+\mathrm{j} 515 \mathrm{kVA}$ in phase $\mathrm{B}$, and $1155+\mathrm{j} 635 \mathrm{kVA}$ in phase $\mathrm{C}$. Loads are categorized as industrial, commercial, and residential (see Table IV). This system has 46 sectionalizing switches and five tie switches (see Fig. 7). Detailed information (e.g., type, parameters, and capacity limits) about each branch and the locations of all switches can be found in [7] and [36]. Moreover, the uncertainty sets of load demands and PV outputs in the worst-case fluctuation scenario, the minimum/maximum bus voltage magnitude, and other simulation parameters (e.g., the convergence tolerance of the C\&CG algorithm) are the same as for the modified IEEE 13-bus network.

In the case of a three-phase fault occurring in branch 45-120 from 16:00 to 20:00 (i.e., $T=4$ and $\Delta d_{t}=1$ ), a power outage will occur after isolating the fault. In this context, the service restoration is conducted using the proposed DRR model and the DDR model. In the following, the initial values of $\Gamma_{\mathrm{L}}$ and $\Gamma_{\mathrm{G}}$ are set to 1 . 
TABLE V

RESTORATION RESULTS FOR TWO RESTORASTION MODELS UNDER THE LARGE TEST SYSTEM

\begin{tabular}{cccccc}
\hline \hline \multirow{2}{*}{ Time Period } & \multirow{2}{*}{ Power Outage $(\mathrm{kW})$} & \multirow{2}{*}{ Model } & Open Line & \multicolumn{2}{c}{ Total Three-phase Restored Power (kW) } \\
\cline { 3 - 6 } 16:00 17:00 & \multirow{2}{*}{1121} & DDR & 39-63, 62-63, 94-95, 116-117 & 1102 & Worst-case Fluctuation \\
& & DRR & $45-47,62-63,95-118,36-117$ & 1096 & 1079 \\
\multirow{2}{*}{ 17:00 18:00 } & \multirow{2}{*}{1168} & DDR & $39-63,62-63,94-95,116-117$ & 1139 & 1113 \\
& & DRR & $45-47,62-63,95-118,36-117$ & 1131 & 1131 \\
18:00 19:00 & \multirow{2}{*}{1425} & DDR & $39-63,62-63,94-95,116-117$ & 1355 & 1319 \\
& \multirow{3}{*}{ 19:00 20:00 } & DRR & $45-47,62-63,95-118,36-117$ & 1348 & 1348 \\
& \multirow{2}{*}{1355} & DDR & $39-63,62-63,94-95,116-117$ & 1305 & 1282 \\
\hline \hline
\end{tabular}

Table V compares the recovery of outage power under the unfluctuating and worst-case fluctuation scenarios. Here, the worst-case fluctuation scenario for the DDR model also refers to the case in which the load demands and PV outputs in each hour remain at their upper bounds during each restoration period. Note that the optimal topology and power recovery are different using these two restoration models. In the case of the unfluctuating condition, the power recovery of the proposed DRR model in each restoration period is slightly lower than that of the conventional DDR model, because the DRR model tries to reduce the optimality slightly to immunize the system against any undesired uncertainties in the bus power injections. However, under the worst-case fluctuation scenario, the DRR strategies in each restoration period are outperformed by the DDR model, which can recover much higher power outages. This is because the excepted load demands (e.g., from bus 45 to bus 51) are rather heavy, and a sharp voltage drop occurs among these buses and those downstream. Thus, several heavy loads must be disconnected if the strategies derived from the DDR model are employed in worst-case scenarios. Under the proposed DRR strategy, branch 45-47 is disconnected and the heavy loads are transferred to another major branch, which avoids the voltage violation.

The following two cases are studied to further validate the advantages of the proposed DRR considering the uncertainty sets of the bus power injections. It is assumed that the uncertainties only vary for the load power demands, and that the uncertainty sets of the PV outputs are the same as those in Subsection III-A.

Case 1 (moderate fluctuation): The interval power demands at each bus in each restoration period are set to $\left[\underline{P}_{\perp}, \bar{P}_{\mathrm{L}}\right]=$ $\left[0.9 P_{\mathrm{L}}, 1.1 P_{\mathrm{L}}\right],\left[\underline{Q}_{\mathrm{L}}, \bar{Q}_{\mathrm{L}}\right]=\left[0.9 Q_{\mathrm{L}}, 1.1 Q_{\mathrm{L}}\right]$.

Case 2 (extreme fluctuation): The interval power demands at each bus in each restoration period are set to $\left[\underline{P}_{L}, \bar{P}_{\mathrm{L}}\right]=$ $\left[1.2 P_{\mathrm{L}}, 1.3 P_{\mathrm{L}}\right],\left[\underline{Q}_{\mathrm{L}}, \bar{Q}_{\mathrm{L}}\right]=\left[1.2 Q_{\mathrm{L}}, 1.3 Q_{\mathrm{L}}\right]$.

MCS is applied to compare the restoration performances of the two models. In each case study, up to 1000 deterministic scenarios (derived from the predefined affine sets) of load demands and PV outputs are generated independently and randomly for restoration. Fig. 8 illustrates the restoration results of the two models under the different fluctuation cases. Because of space limitations, only the restoration results in the third time period (from 18:00-19:00) are illustrated. Similar results are obtained in the other time periods.

The variability of load demands clearly affects the solution

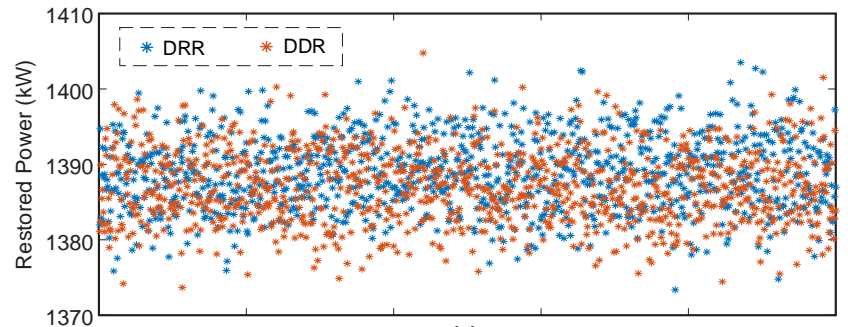

(a)

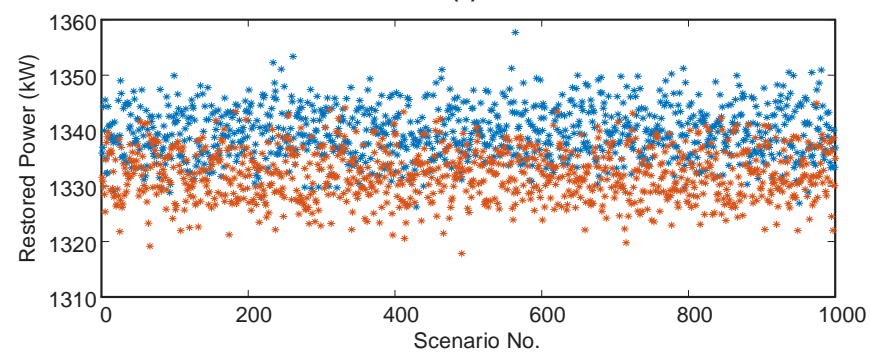

(b)

Fig. 8. Comparison of restored power based on 1000 MCS for (a) Case 1 and (b) Case 2.

TABLE VI

CPU TIME OF THESE TWO RESTORATION MODELS UNDER DIFFERENT ELECTRICAL NETWORKS

\begin{tabular}{ccc}
\hline \hline \multirow{2}{*}{ Restoration Model } & \multicolumn{2}{c}{ CPU time (s) } \\
\cline { 2 - 3 } & IEEE 13-bus & IEEE 123-bus \\
\hline DDR & 0.78 & 27.19 \\
DRR & 20.69 & 1605.93 \\
\hline \hline
\end{tabular}

outcomes of the two models. As the deviation values increase, the restored power of both models decreases and their difference increases accordingly. For instance, the average power restored by DDR and DRR in Case 1 is $1391.97 \mathrm{~kW}$ and $1389.56 \mathrm{~kW}$, respectively, whereas in Case 2, the corresponding values are $1331.63 \mathrm{~kW}$ and $1343.85 \mathrm{~kW}$, respectively. Nevertheless, the proposed DRR model typically outperforms the DDR model in both cases in terms of handling randomness, especially in the extreme case. This is attributed to the fact that the robust method is less sensitive to uncertainty, and can recover more power outage under highly variable bus power injections.

Finally, the computational burden of the two restoration models is compared. Table VI summarizes the CPU time required to restore power outages in the small-scale/large-scale test systems. The robust DRR model requires more time in total to complete the restoration process than the deterministic model, because the C\&CG algorithm in each robust model 


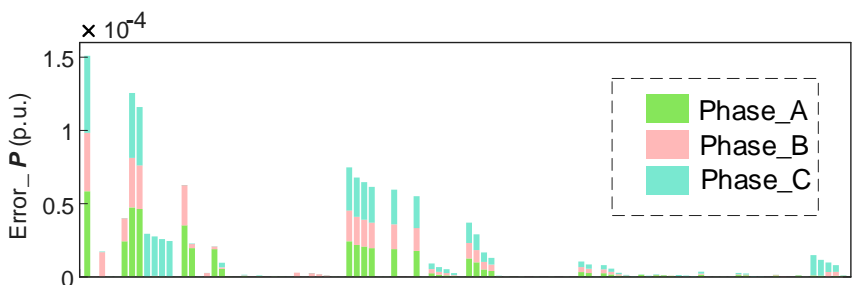

(a)

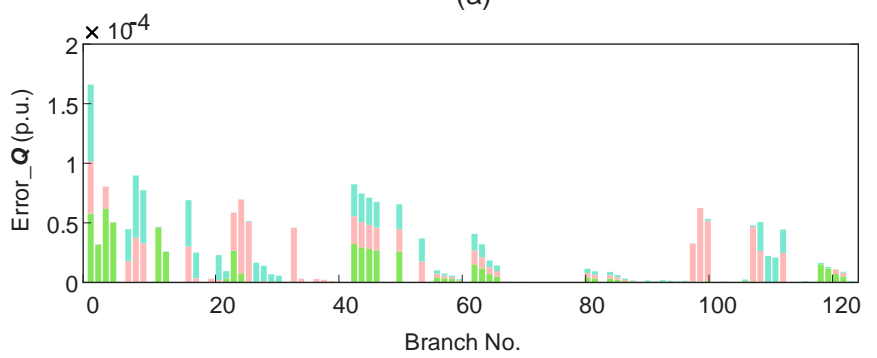

(b)

Fig. 9. Error profiles of power flows (shown in stacked form) in the first time period for (a) active and (b) reactive power flows.

solves sub and master problems iteratively until the convergence tolerance is satisfied. Larger distribution networks significantly increase the number of total derivative variables and constraints in the C\&CG algorithm, and thus increase the computational time. Although the C\&CG algorithm consumes significantly more time as the problem size increases, the proposed DRR model is to be used offline, and so the computational efficiency is acceptable. Nevertheless, many speedup algorithms [25] could be used to improve the computational efficiency of DRR.

\section{Discussion of the Linear Approximation}

In Subsection II-B, an optimal equidistant piecewise-linear algorithm was introduced to convert the original MIQCP model of the restoration into an MILP problem. In the two case studies discussed above, the maximum linear approximation error of each phase of the active and reactive power flows at each time period are predefined as $\mathrm{C}=0.001$ (p.u.), and then 112 equal-length segments are generated. At this point, the accuracy of the linear approximation approach is of interest. The following absolute error formulations of active and reactive power flows are considered:

$$
\left\{\begin{array}{c}
\text { error_} P=\left|P_{i k, \mathrm{~A}}^{\varphi, t}-P_{i k,+}^{\varphi, t}-P_{i k,-}^{\varphi, t}\right| \\
\text { error_}_{-} \boldsymbol{Q}=\left|Q_{i k, \mathrm{~A}}^{\varphi, t}-Q_{i k,+}^{\varphi, t}-Q_{i k,-}^{\varphi, t}\right|
\end{array}\right.
$$

where $P_{i k, \mathrm{~A}}^{\varphi, t}$ and $Q_{i k, \mathrm{~A}}^{\varphi, t}$ are the true values of the active and reactive power flows, respectively.

The error profiles of active and reactive power flows derived from the linear approximation approach in the first time period are illustrated in Fig. 9. The simulation results from other time periods give similar results. The deviations in the linear models for each phase are lower than the given maximum values and small enough to be negligible. In this context, it can be concluded that the optimal equidistant piecewise-linear algorithm in the proposed DRR model is accurate.

\section{CONCLUSIONS}

This paper has presented a DRR framework for restoring power outages considering uncertainty scenarios in unbalanced distribution networks. To improve the performance of DRR, reasonable interval modeling schemes of DG outputs have been presented. Next, owing to the dual-transformation and optimal equidistant piecewise-linear algorithms, the formulation was converted to an MILP problem with a bi-level function, and solutions were obtained using the C\&CG algorithm. The results of numerical experiments have validated the superiority of the proposed DRR model in comparison with a deterministic model for restoration performance under fluctuating DG outputs and time-varying load demands. This is useful in providing insights into self-healing systems for the uncertainties of power distribution networks. In future work, aspects such as the black-start ability of DG, the restoration of isolated micro-grids, and the DRR model for weakly meshed distribution networks could prove to be of value.

\section{REFERENCES}

[1] C. Chen, J. Wang, and D. Ton, "Modernizing distribution system restoration to achieve grid resiliency against extreme weather events: an integrated solution,” Pro. IEEE, vol. 105, no. 7, pp. 1267-1288, Jul. 2017.

[2] M. Eriksson, M. Armendariz, O. O. Vasilenko, A. Saleem, and L. Nordstrom, "Multiagent-based distribution automation solution for self-healing grids," IEEE Trans. Ind. Electron., vol. 62, no. 4, pp. 26202628, Apr. 2015.

[3] S. A. Arefifar, Y. -A.-R. I. Mohamed and T. El-Fouly, "Optimized multiple microgrid-based clustering of active distribution systems considering communication and control requirements," IEEE Trans Ind. Electron., vol. 62, no. 2, pp. 71-723, Feb. 2015.

[4] X. Yu and Y. Xue, "Smart grids: a cyber-physical systems perspective," Pro. IEEE, vol. 14, no. 5, pp. 1058-1070, May 2016.

[5] J. Xu, Z. Wu, X. Yu and C. Zhu, "Robust faulted line identification in power distribution networks via hybrid state estimator," IEEE Trans. Ind. Informat., vol. 15, no. 9, pp. 5365-5377, Sept. 2019.

[6] Y.-J. Kim, J. Wang, and X. Lu, “A framework for load service restoration using dynamic change in boundaries of advanced microgrids with synchronous-machine DGs," IEEE Trans. Smart Grid, vol. 9, no. 4, pp. 3676-3690, Jul. 2018.

[7] Z. Wang, J. Wang and C. Chen, , "A three-phase microgrid restoration model considering unbalanced operation of distributed generation," IEEE Trans. Smart Grid, vol. 9, no. 4, pp. 3594-3604, Jul. 2018.

[8] J. Xu, Z. Wu, X. Yu, Q. Hu and X. Dou, "An interval arithmetic-based state estimation framework for power distribution networks," IEEE Trans. Ind. Electron., vol. 66, no. 11, pp. 8509-8520, Nov. 2019.

[9] X. Chen, W. Wu and B. Zhang, "Robust restoration method for active distribution networks," IEEE Trans. Power Syst., vol. 31, no. 5, pp. 40054015, Sept. 2016.

[10] H.-C. Kuo and Y.-Y. Hsu, "Distribution system load estimation and service restoration using a fuzzy set approach," IEEE Trans. Power Del., vol. 8, no. 4, pp. 1950-1957, Oct. 1993.

[11] S.-J. Lee, S.-I. Lim, and B.-S. Ahn, "Service restoration of primary distribution systems based on fuzzy evaluation of multi-criteria," IEEE Trans. Power Syst., vol. 13, pp. 1156-1163, Aug. 1998.

[12] D. S. Popovic and Z. N. Popovic, "A risk management procedure for supply restoration in distribution networks," IEEE Trans. Power Syst., vol. 19, no. 1, pp. 221-228, Feb. 2004.

[13] W.-H. Chen, M.-S. Tsai, and H.-L. Kuo, "Distribution system restoration using the hybrid fuzzy-grey method," IEEE Trans. Power Syst., vol. 20, no. 1, pp. 199-205, Feb. 2005.

[14] A. M. Prostejovsky, O. Gehrke, A. M. Kosek, T. Strasser and Henrik W. Bindner, "Distribution line parameter estimation under consideration of measurement tolerances," IEEE Trans. Ind. Informat., vol. 12, no. 2, pp. 726-735, Apr. 2015.

[15] A.K.-Fard and T. Niknam, "Optimal distribution feeder reconfiguration for reliability improvement considering uncertainty," IEEE Trans. Power Del., vol. 29, no. 3, pp. 1344-1353, Jun. 2014.

[16] H. Gao, Y. Chen, Y. Xu and C. Liu, "Resilience-oriented critical load restoration using microgrids in distribution systems," IEEE Trans. Smart Grid, vol. 7, no. 6, pp. 2837-2848, Nov. 2016. 
[17] X. Huang, Y. Yang, and G. A. Taylor, "Service restoration of distribution systems under distributed generation scenarios," CSEE J. Power Energy Syst., vol. 2, no. 3, pp. 43-50, Sep. 2016.

[18] L. T. Marques, A. C. B. Delbem and J. B. A. London, "Service restoration with prioritization of customers and switches and determination of switching sequence,” IEEE Trans. Smart Grid, vol. 9, no. 3, pp. 23592370, May 2018.

[19] A. Sharma, D. Srinivasan, and A. Trivedi, "A decentralized multi-agent approach for service restoration in uncertain environment," IEEE Trans. Smart Grid, vol. 9, no. 4, pp. 3394-3405, Jul. 2018.

[20] Y. Zhao, Z. Lin, Y. Ding, Y. Liu, L. Sun, and Y. Yan, "A model predictive control based generator start-up optimization strategy for restoration with microgrids as black-start resources,” IEEE Trans. Power Syst., vol. 33, no. 6, pp. 7189-7203, Nov. 2018.

[21] Y. Wang, Y. Xu, J. He, C. C. Liu, K. P. Schneider, M. Hong, and D. T. Ton, "Coordinating multiple sources for service restoration to enhance resilience of distribution systems," IEEE Trans. Smart Grid, vol. 10, no. 5, pp. 5781-5793, Sept. 2019.

[22] H. Pan, X. Jing, W. Sun and H. Gao, "A Bioinspired Dynamics-Based Adaptive Tracking Control for Nonlinear Suspension Systems,” IEEE Trans. Control Syst. Technol., vol. 26, no. 3, pp. 903-914, May 2018.

[23] Z. Wang, C. Shen, Y. Xu, F. Liu, X. Wu and C. Liu, "Risk-limiting load restoration for resilience enhancement with intermittent energy resources," IEEE Trans. Smart Grid, vol. 10, no. 3, pp. 2507-2522, May 2019.

[24] V. Loia, V. Terzija, A. Vaccaro and P. Wall, "An affine-arithmetic-based consensus protocol for smart-grid computing in the presence of data uncertainties,” IEEE Trans. Ind. Electron., vol. 62, no. 5, pp. 2973-2982, May 2015.

[25] K. Chen, W. Wu, B. Zhang, and H. Sun, "Robust restoration decisionmaking model for distribution networks based on information gap decision theory,” IEEE Trans. Smart Grid, vol. 6, no. 2, pp. 587-597, Feb. 2015.

[26] Y. Xie, X. Chen, Q. Wu and Q. Zhou, “Second-order conic programming model for load restoration considering uncertainty of load increment based on information gap decision theory," Int. J. Electri. Power Energy Syst., vol. 105, pp. 151-158, Feb. 2019.

[27] S. Chen, Z. Wei, G. Sun, K. W. Cheung, D. Wang and H. Zang, "Adaptive robust day-ahead dispatch for urban energy systems," IEEE Trans. Ind. Electron., vol. 66, no. 2, pp. 1379-1390, Feb. 2019.

[28] C. Huang, D. Yue, J. Xie, Y. Li and K. Wang, "Economic dispatch of power systems with virtual power plant based interval optimization method," CSEE J. Power Energy Syst., vol. 2, no. 1, pp. 74-80, Mar. 2016.

[29] Y. Xiang, J. Liu, and Y. Liu, "Robust energy management of microgrid with uncertain renewable generation and load," IEEE Trans. Smart Grid, vol. 7, no. 2, pp. 1034-1043, Mar. 2016.

[30] A. Khosravi, S. Nahavandi, D. Creighton and A. F. Atiya, "A lower upper bound estimation method for construction of neural network based prediction intervals," IEEE Trans. Neural Netw., vol. 22, no. 3, pp. 337346, Mar. 2011.

[31] C. Liu and Y. Hsu, "Design of a self-tuning PI controller for a STATCOM using particle swarm optimization,” IEEE Trans. Ind. Electron., vol. 57, no. 2, pp. 702-715, Feb. 2010.

[32] R. Perez-Guerrero, G. T. Heydt, N. J. Jack, B. K. Keel, and A. R. Castelhano, "Optimal restoration of distribution systems using dynamic programming,” IEEE Trans. Power Del., vol. 23, no. 3, pp. 1589-1596, Jul. 2008.

[33] A. Anwar, A. N. Mahmood, and Z. Tari, "Ensuring data integrity of OPF module and energy database by detecting changes in power flow patterns in smart grids," IEEE Trans. Ind. Informat., vol. 13, no. 6, pp. 3299-3311, Dec. 2017.

[34] H. Haghighat and B. Zeng, "Distribution system reconfiguration under uncertain load and renewable generation," IEEE Trans. Power Syst., vol. 31, no. 4, pp. 2666-2675, Jul. 2016.

[35] A. Rantzer and M. Johansson, "Piecewise linear quadratic optimal control,” IEEE Trans. Autom. Control, vol. 45, no. 4, pp. 629-637, Apr. 2000.

[36] Resources. IEEE PES AMPS DSAS Test Feeder Working Group. [Online]. Available: http://ewh.ieee.org/soc/pes/dsacom/testfeeders/, Revised, Feb. 2014.

[37] R. Yan, T. K. Saha, P. Meredith and A. Ananth, "Megawatt-scale solar variability study: an experience from a 1.2 MW photovoltaic system in Australia over three years," IET Renew. Power Gener., vol. 10, no. 8, pp. 1229-1236, 2016.

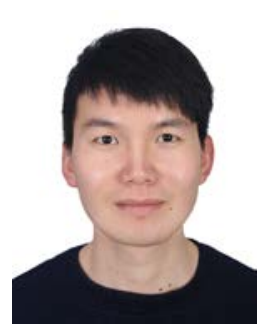

Junjun Xu (S’17- M'19) received the B.S. degree in power system and its automation from Nanjing Institute of Technology, Nanjing, China, in 2012, the M.S. degree in agricultural electrification and automation from the School of Electrical and Information Engineering, Jiangsu University, Zhenjiang, China, in 2015, and the Ph.D. degree in electrical engineering from Southeast University, Nanjing, China, in 2019. He joined the College of Automation, College of Artificial Intelligence, Nanjing University of Posts and Telecommunications (NUPT), Nanjing, China, in Dec. 2019.

His research interests include distribution network state estimation, self-healing control and uncertainty modeling approaches.

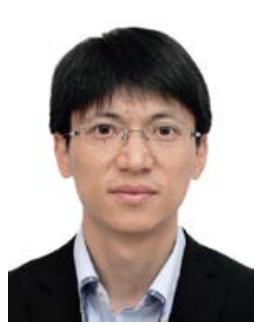

Zaijun Wu (M'12) received the B.Eng. degree in power system and its automation from the Hefei University of Technology, Hefei, China, in 1996, and the Ph.D. degree in electrical engineering from Southeast University, Nanjing, China, in 2004. He was a Visiting Scholar at the Ohio State University, USA, from 2012 to 2013. He is currently a Professor in electrical engineering with the School of Electrical Engineering, Southeast University.

His research interests include micro-grid, active distribution network, and power quality. He is the author or coauthor of more than 130 referred journal papers, and a reviewer of several journals.

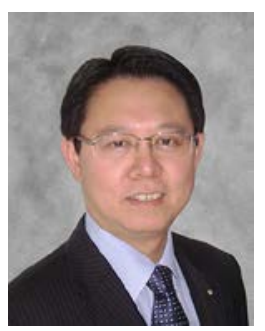

Xinghuo Yu (M'92-SM'98-F'08) received the B.Eng and M.Eng degrees in electrical engineering from the University of Science and Technology of China, Hefei, China, in 1982 and 1984, and the Ph.D. degree in automatic control from Southeast University, Nanjing, China, in 1988, respectively.

He is an Associate Deputy Vice-Chancellor and a Distinguished Professor in control science and engineering of RMIT University (Royal Melbourne Institute of Technology), Melbourne, Victoria, Australia. His research interests include variable structure and nonlinear control, complex and intelligent systems, and smart energy systems.

Prof. Yu has received a number of awards and honors for his contributions, including the 2018 Australasian Artificial Intelligence Distinguished Research Contribution Award, the 2018 M. A. Sargent Medal of Engineers Australia, the 2013 Dr.-Ing. Eugene Mittelmann Achievement Award of the IEEE Industrial Electronics Society, and the 2012 IEEE Industrial Electronics Magazine Best Paper Award. He was the President (2018-2019) of the IEEE Industrial Electronics Society. He was named a Highly Cited Researcher by Clarivate Analytics (formerly Thomson Reuters) from 2015 to 2018.

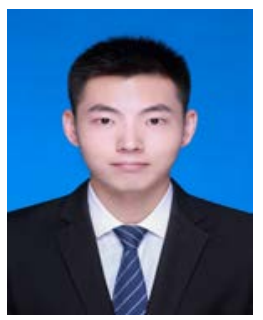

Sheng Cheng received the B.S. degree in electrical engineering and automation from Southwest Jiaotong University, Chengdu, China, in 2016, and the M.S. degree in electrical engineering from the School of Electrical Engineering, Southeast University, Nanjing, China, in 2019. He is currently working in China Energy Engineering Group Jiangsu Power Design Institute Co., Ltd, Nanjing, China.

His research interests include optimal operation system for substation. and protection control of power system and secondary 


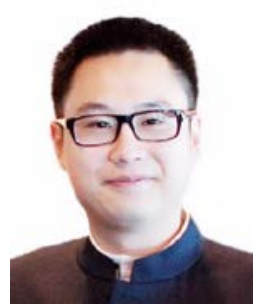

Qinran Hu (S'11-M'15) received the B.S. degree from Chien-Shiung Wu Honors College, Southeast University (China) in 2010, and the M.S. and Ph.D. degrees from the University of Tennessee, Knoxville, TN, USA in 2013 and 2015, respectively. He was a postdoc fellow in Harvard University, Cambridge, MA, USA from 2015 to 2018. He joined the School of Electrical Engineering, Southeast University, Nanjing, China, in Oct. 2018.

His research interests include power system optimization, demand aggregation, and electricity market.

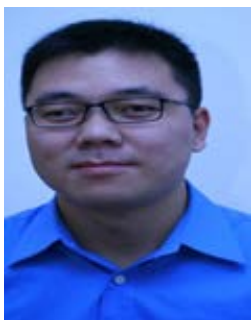

Qiuwei Wu (M'08-SM'15) received the Ph.D. degree in power system engineering from Nanyang Technological University, Singapore, in 2009. He was a Senior Research and Development Engineer with VESTAS Technology R\&D Singapore Pte, Ltd., from March 2008 to October 2009. He has been with the Department of Electrical Engineering, Technical University of Denmark since Nov. 2009 (PostDoc Nov. 2009 - Oct. 2010, Assist Prof. Nov. 2010 - Aug. 2013, Assoc Prof Sept. 2013 - ). In 2012, he was a Visiting Scholar with the Department of Industrial Engineering and Operations Research, University of California, Berkeley, for three months funded by Danish Agency for Science, Technology and Innovation, Denmark. He was a Visiting Scholar with the Harvard China Project, School of Engineering and Applied Sciences, Harvard University from 2017 to 2018.

His research interests are operation and control of power systems with high penetration of renewables, including wind power modeling and control, active distribution networks, and operation of integrated energy systems. He is the Deputy-Editor-In-Chief of the International Journal of Electrical Power and Energy Systems. He is an Editor of the IEEE Transactions on Smart Grid and IEEE Power Engineering Letters. He is the Regional Editor for Europe of the IET Renewable Power Generation, a Subject Editor of IET Generation, Transmission and Distribution and an Associate Editor of the Journal of Modern Power Systems and Clean Energy. 